\title{
Short naphthalene organophosphonate linkers to microporous
}

\section{frameworks}

\author{
Aysun Bulut, ${ }^{[b, c]}$ Yunus Zorlu, ${ }^{[\mathrm{d}]}$ Michael Wörle, ${ }^{[\mathrm{e}]}$ Ahmet Çetinkaya, ${ }^{[\mathrm{fl}}$ Hüseyin Kurt, ${ }^{[\mathrm{g}]}$ Benjamin Tam, ${ }^{[\mathrm{h}]}$ \\ Özgür Yazaydın, ${ }^{[\mathrm{h}]}$ Jens Beckmann ${ }^{[\mathrm{i}]}$ and Gündoğ Yücesan ${ }^{*}{ }^{\text {[a] }}$
}

\begin{abstract}
We report three novel 3D porous metalorganophosphonate metal organic frameworks (MOFs) $\left[\left\{\mathrm{Cu}\left(4,4{ }^{\prime}\right.\right.\right.$ bpy $\left.)_{0.5}\left(1,4-N D P A-H_{2}\right)\right] \quad(\mathbf{1}), \quad\left[\left\{\mathrm{Cu}_{2}\left(4,4{ }^{\prime}-\text {-bpy }\right)_{0.5}\right\}(1,4-\mathrm{NDPA})\right] \quad(\mathbf{2})$ and $[\{\mathrm{Cu}(4,4$ '-bpy)\}(2,6-NDPA-H 2$)](3)$ constructed using the structurally rigid 1,4-naphthalenediphosphonic acid $\left(1,4-N D P A-H_{4}\right)$ and 2,6naphthalenediphosphonic acid (2,6-NDPA- $\left.\mathrm{H}_{4}\right) .1$ and 2 exhibit the highest surface areas obtained using the structurally rigid and short aromatic organophosphonate linkers. The compound $\mathbf{1}$ has been further analyzed by TGA and Quantum Design PPMS vibrating sample magnetometer.
\end{abstract}

The practice of metal-organophosphonates (MOPs) to obtain predictable pore sites is an important goal yet to be achieved by the MOF chemists as porous MOPs are expected to introduce a wider palette of applications and robustness into the future

[a] Dr. G. Yücesan

Department of Food Chemistry and Toxicology,

Technische Universitat Berlin

Gustav-Meyer-Allee 25, 13355 Berlin, Germany

E-mail: yuecesan@tu-berlin.de

[b] A. Bulut

Faculty of Pharmacy,

Istanbul Kemerburgaz University,

Bakirkoy, Istanbul, Turkey

[c] A. Bulut

Department of Chemistry

Bogazici University

34342 Istanbul, Turkey

[d] Prof. Y. Zorlu

Department of Chemistry

Gebze Technical University

P.O. Box 141 Gebze, 41400 Kocaeli, Turkey

[e] Dr. M. Wörle

Laboratory of Inorganic Chemistry,

ETH Zurich, $\mathrm{HCl} \mathrm{H103} \mathrm{Vladimir-Prelog-Weg} \mathrm{1,} \mathrm{CH-8093,}$ Switzerland

[f] A. Cetinkaya

Department of Bioengineering

Yildiz Technical University,

Istanbul, Turkey

[g] Prof. H. Kurt

Department of Engineering Physics,

Istanbul Medeniyet University,

Istanbul, Turkey

[h] B. Tam, Prof. A. Ö. Yazaydın

Department of Chemical Engineering

University College London

London WC1E 7JE, United Kingdom

[i] Prof. J. Beckmann

Institut für Anorganische Chemie und Kristallographie

Universitat Bremen

Leobener Strase, 28359 Bremen, Germany

Supporting information for this article is given via a link at the end of the document.
MOFs. ${ }^{[1]}$ In order to achieve the synthesis of predictable porous networks in MOPs, metal cluster or simple one-dimensional secondary building unit (SBU) approach should be developed with precise control of the angle between the bridging ligands and the SBU core. ${ }^{[2]}$ The structural control in MOF synthesis has been partially achieved with the carboxylate linkers. ${ }^{[3]}$ The major difference between the organophosphonate and carboxylate linkers is the structure of the adhesive functional unit, where carboxylates exhibit simpler trigonal planar connectivity with precise binding modes while organophosphonates are tetrahedral with numerous possible binding and protonation modes. ${ }^{[4]}$ Because of the richness of the binding modes, MOPs usually produce uncontrollable metal oxide condensations in one, two and three-dimensions to produce highly dense pillared-layered structures. ${ }^{[5]}$ Another parameter to achieve the goal of establishing the pore sites in MOP chemistry is the regulation of the binding modes of the organophosphonate units while tuning the angle between the organic linkers. ${ }^{[5 b]}$ Trigonal planar tritopic aromatic organophosphonates and the tetrahedral organophosphonates have been shown to prove this hypothesis to produce predictable voids, as their bridging arms are already separated by angles of $120^{\circ}$ and $108^{\circ}$ around triazine, methane, silane and adamantane cores. ${ }^{[6]}$ The well separated phosphonate arms directed the formation of metal clusters to form void spaces. Such void spaces haven't been observed with the linear aromatic organophosphonates yet. ${ }^{[1]}$

As seen in the tetratopic and tritopic long branched aromatic organophosphonates, the geometry, orientation and binding modes of the bridging ligands are the most significant factors determining the final structure and the applications of the metal organic solids. 1,4-benzenediphosphonic acid is the most used linear aromatic organophosphonate linker in the literature. ${ }^{[7]}$ The unsystematic study of this ligand by various research groups have produced random interesting properties with dense lamellar and pillared layered structures while the MOFs constructed using the structurally analogous 1,4-benzenedicarboxylic acid produced large surface areas between 2600 to $2900 \mathrm{~m}^{2} / \mathrm{g}^{[8]}$ On the other hand, each of the reported structures with 1,4phenyldiphosphonic acid offered interesting proton conductivity, ion exchange and magnetic properties, but to date no porous platforms using the 1,4-benzenediphosphonic acid has been achieved. ${ }^{[7]}$ During the course of this study, we used structurally similar 1,4-naphthalenediphosphonic acid. We have further explored the effect of the additional phenyl group and the increased structural rigidity.

The naphthalene chemistry has been a very popular research area since the beginning of the $20^{\text {th }}$ century. There have been innumerous articles published in the literature investigating the naphthalene core. One of the interesting features of naphthalene is its structural rigidity, which has been used as a center for a bridging ligand to construct functional MOFs. Most notably, 2,6naphthalenedicarboxylic acid lead the synthesis of IRMOF- 8 by 
Yaghi. ${ }^{[9]}$ 1,4-naphthalenedicarboxylic acid lead the synthesis of MOF-49 and 50. ${ }^{[10]}$ On the other hand, introduction of the phosphonate functional group around the naphthalene scaffold was not achieved until very recently. We have just published the structure and binding modes of 2,6-naphthalenediphosphonic acid (2,6-NDPA- $\left.\mathrm{H}_{4}\right) .{ }^{[5 b]}$ 1,4-naphthalenediphosphonic acid (1,4NDPA- $\mathrm{H}_{4}$ ) was published in 2012. ${ }^{[11]}$ During the course of this study, we have used $1,4-\mathrm{NDPA}-\mathrm{H}_{4}$, which is structurally similar to 1,4-benzenediphosphonic acid with an additional phenyl ring making it structurally more rigid and explored its potential to form void spaces in comparison with the parent 1,4benzenediphosphonic acid. We have also altered the reaction conditions to apply more control over the binding modes of the organophosphonate units to regulate the structural diversity and finally used 2,6-NDPA- $\mathrm{H}_{4}$ to further engineer the binding angles of the potential of organophosphonate linkers. In addition to engineering the organophosphonate structure, we have also employed the auxiliary organonitrogen compound 4,4'-bipyridine $\left(4,4^{\prime}\right.$-bpy) to control the coordination environment of copper ions and to introduce additional bridging potential. Here we report the three-dimensional MOFs $\left[\left\{\mathrm{Cu}\left(4,4^{\prime}-\mathrm{bpy}\right)_{0.5}\left(1,4-\mathrm{NDPA}-\mathrm{H}_{2}\right)\right]\right.$ (1), $\left[\left\{\mathrm{Cu}_{2}\left(4,4^{\prime}-\mathrm{bpy}\right)_{0.5}\right\}(1,4-\mathrm{NDPA})\right](2)$ and $\left[\left\{\mathrm{Cu}\left(4,44^{\prime}-\mathrm{bpy}\right)\right\}(2,6-\mathrm{NDPA}-\right.$ $\left.\mathrm{H}_{2}\right)$ ] (3) constructed with the structurally rigid 1,4- and 2,6naphthalenediphosphonic acid units. The structures of 1, 2 and 3 (Figs. S1-S3) have been characterized by single crystal X-ray diffraction. The compounds have been further analyzed with TGA and their magnetic analysis was performed using Quantum Design PPMS vibrating sample magnetometer. BET surface areas of $\mathbf{1}$ and $\mathbf{2}$ were derived from $\mathrm{N}_{2}$ adsorption isotherms at $77 \mathrm{~K}$ which were predicted by Monte Carlo simulations in the grand canonical ensemble (see SI for the details of simulations).

The compounds 1, 2 and $\mathbf{3}$ have similar 3D structural patterns. Each of the structures are composed of Cu-O-P-O chains and these chains are connected to form the $3 \mathrm{D}$ frameworks via the 1,4-NDPA- $\mathrm{H}_{4}$ and the $4,4^{\prime}$-bpy linkers. One common feature of these three compounds is that the 4,4'-bpy linkers naphthalenediphosphonates are always parallel to each other in each of the structures. The size of pore sites in $\mathbf{1}$ and $\mathbf{2}$ is established with respect to the angle between the naphthalenediphosphonate and the 4,4'-bpy units, which are connecting the $\mathrm{Cu}-\mathrm{O}-\mathrm{P}-\mathrm{O}$ chains to form the three-dimensional framework. Therefore, unlike the dense pillared layered structures where all the bridging ligands are parallel to each other, in this case $4,4^{\prime}$-bpy units and $1,4-\mathrm{NDPA}^{-} \mathrm{H}_{4}$ are not parallel to each other. The copper coordination is square pyramidal in $\mathbf{1}$, distorted octahedral in 2 and octahedral in 3. 4,4'-bipyridine and 1,4naphtalenediphosphonic acid linkers have nearly $90^{\circ}$ angle producing rectangular voids in $\mathbf{2}$ making the chain structure in $\mathbf{2}$ a 1D secondary building unit that could potentially produce isoreticular expansions to give rectangular voids in different sizes and shapes.

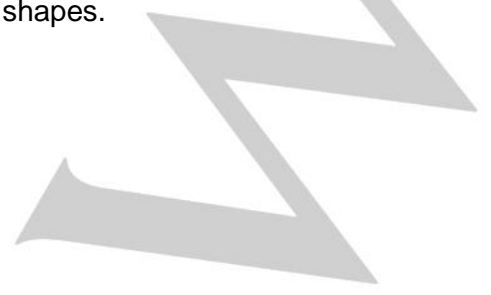

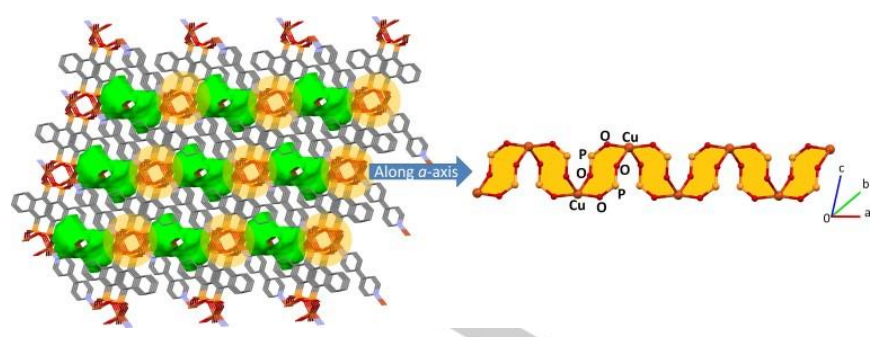

Figure 1. The $3 \mathrm{D}$ structure of 1 , showing the voids highlighted in green within the framework and 1D SBU composed of edge sharing Cu-O-P-O-Cu-O-P-O rings.

As seen in Fig. 1, the chain structure in compound 1 is composed of 8 membered edge sharing Cu-O-P-O-Cu-O-P-O rings along aaxis with square pyramidal $\mathrm{Cu}(\mathrm{II})$ and fully deprotonated phosphonate ends. These chains are connected to form the 3D MOF structure via 4,4'- bpy and 1,4-NDPA- $\mathrm{H}_{4}$ linkers with an angle of nearly $30^{\circ}$ with respect to the a-axis. The simplicity of the chain structure in $\mathbf{1}$ indicates that it could be facilitated as onedimensional secondary building unit forming larger voids with improved tether lengths in the future.

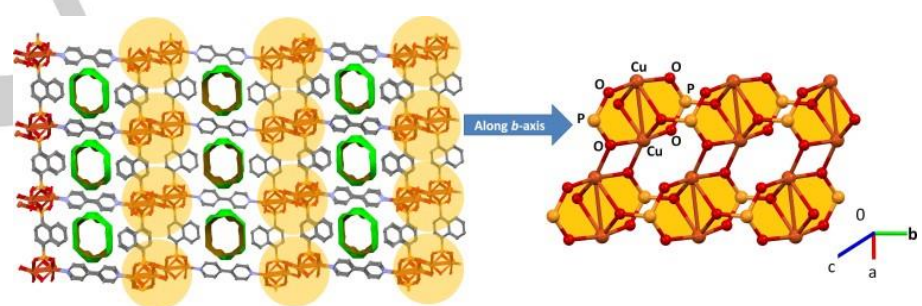

Figure 2. The $3 D$ structure of $\mathbf{2}$, showing the voids highlighted in green within the framework and 1D SBU with edge sharing Cu-O-P-O- rings, and the central bridging $\left\{\mathrm{Cu}_{2} \mathrm{O}_{2}\right\}$ rhombi.

As seen in Fig. 2, the chain structure in compound 2 is composed of two identical $\left\{\mathrm{Cu}_{2} \mathrm{O}_{2}\right\}$ rhombi with alternating short-long $\mathrm{Cu}-\mathrm{O}$ distances. The inner $\mathrm{Cu}$ (II) centers, which don't coordinate to the 4,4'-bpy units make another $\left\{\mathrm{Cu}_{2} \mathrm{O}_{2}\right\}$ rhombus with almost identical $\mathrm{Cu}-\mathrm{O}$ distances. These three adjacent rhombi are connected together via 8 membered Cu-O-P-O-Cu-O-P-O rings to give the basic structure of the one-dimensional chain. The chains in 2 were connected by 4,4'-bpy and 1,4naphthalenediphosphonic acid units with an angle of near 90 degrees with respect the $x$ axis to form the rectangular voids. As seen in Fig. 3, compound 3 has the same 8 membered chain structure observed in 1. Unlike the square pyramidal coppers in $\mathbf{1}$, the copper centers in $\mathbf{3}$ are octahedral. Therefore, the structural difference between 1 and $\mathbf{3}$ is originating from the octahedral copper coordination. In compound one 4,4-bipyridine coordinates on the basal plane while in 3, two 4,4'-bipyridine units coordinate at the apical positions. As a result of the additional 4,4'-bpy coordination around the 8 membered Cu-O-P-O-Cu-O-P-O rings along $a$-axis, the structure of $\mathbf{3}$ is much denser compared to $\mathbf{1}$. Fig. 4 shows simulated $\mathrm{N}_{2}$ isotherm of 1 and 2 at $77 \mathrm{~K}$, which demonstrate rapid filling of relatively narrow pores. BET surface 
areas calculated from the simulated isotherms of $\mathbf{1}$ and $\mathbf{2}$ were $229.2 \mathrm{~m}^{2} \mathrm{~g}^{-1}$ and $132.1 \mathrm{~m}^{2} \mathrm{~g}^{-1}$, respectively. ${ }^{[12]}$

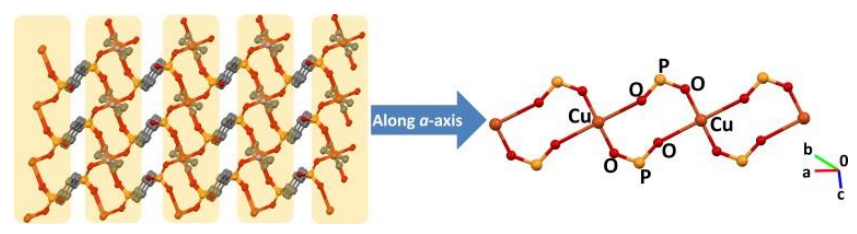

Figure 3. The 3D framework of 3 , edge sharing Cu-O-P-O-Cu-O-P-O rings.
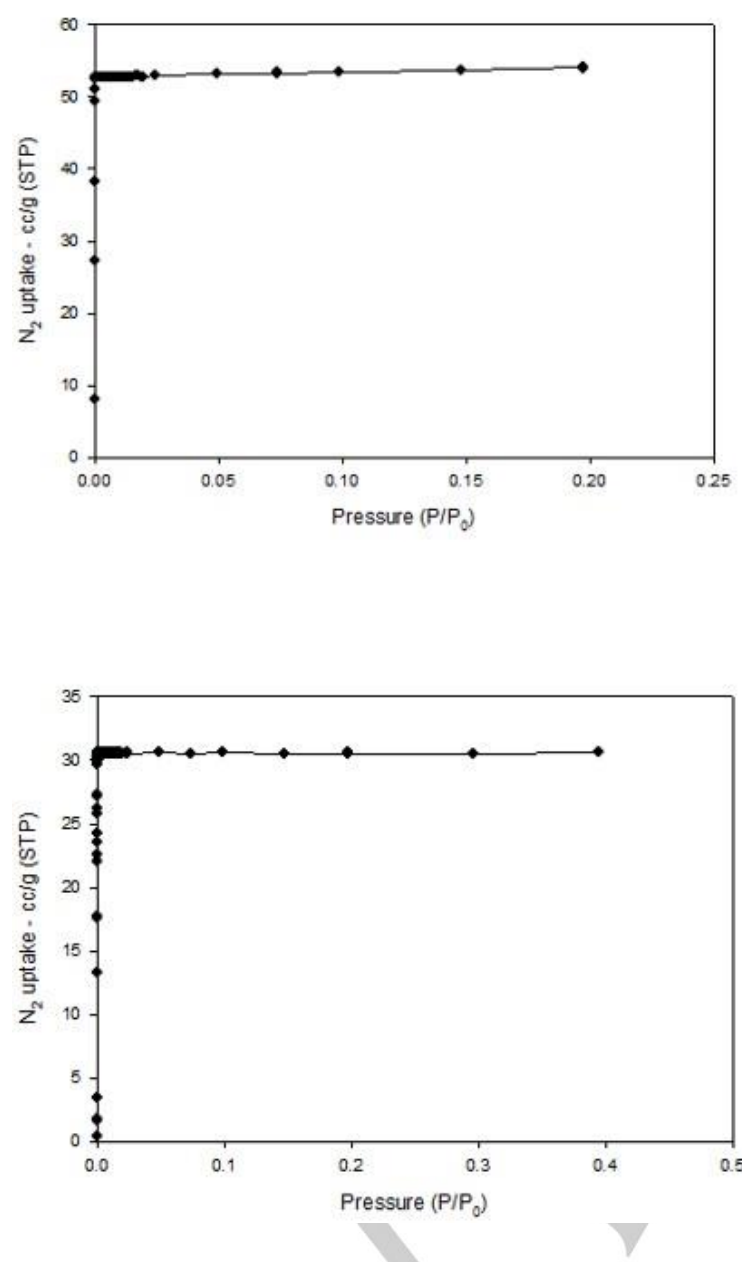

Figure 4. Simulated $\mathrm{N}_{2}$ isotherms at $77 \mathrm{~K}$ in 1 (top) and 2 (bottom).

The magnetization measurements were made using a Quantum Design PPMS vibrating sample magnetometer. The sample 1 shows paramagnetic behavior (see Fig. 5) at room temperature with a paramagnetic susceptibility $\chi=1.26 \times 10^{-5} \mathrm{emu} / \mathrm{gr}$ Oe. Magnetization measurements from $300 \mathrm{~K}$ to $10 \mathrm{~K}$ were made in the field cooling mode at a constant magnetic field of $500 \mathrm{Oe}$. The sample does not show any magnetic phase change during cooling. The noisy data seen at temperatures above $170 \mathrm{~K}$ is due to the movement of small particles during measurement and the very small magnetic moment. To confirm this, we have also measured $\mathrm{M}$ vs. $\mathrm{H}$ at $200 \mathrm{~K}$ and did not see any hysteresis. The Curie Constant $\mathrm{C}=13160 \mathrm{AK} / \mathrm{Tm}$ was found by applying Curie Law $(\mathrm{M}=\mathrm{CB} / \mathrm{T})$ to temperature dependent magnetization data in the 10-150K range.
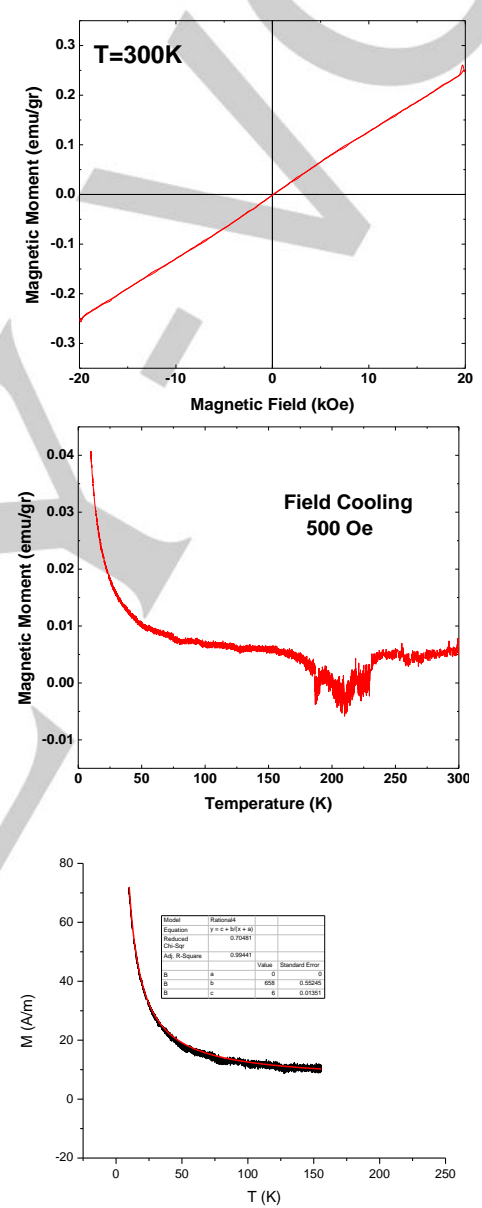

Figure 5. (a) Magnetization measurement of 1showing paramagnetic behavior at $300 \mathrm{~K}$ (b) Magnetization vs. temperature with a dc field cooling from $300 \mathrm{~K}$ at 500 Oe (c) Curie Law fitting of the curve from 10-150K.

Thermogravimetric analysis was conducted only for the sample 1 with a rate of $10^{\circ} \mathrm{C} / \mathrm{min}$. The yield for 2 and $\mathbf{3}$ was very low to conduct the thermogravimetric analysis. Organonitrogen ligands usually start decomposing earlier. Therefore, the decomposition of the first $21.7 \%$ weight loss starting at ca. $375^{\circ} \mathrm{C}$ is associated with the 4,4'-bpy unit ( $19 \%$ calculated). The second $24,1 \%$ weight loss start at ca. $450{ }^{\circ} \mathrm{C}$ is in accordance with the previously reported metal-organophosphonate decomposition temperatures 
and it is associated with the organic components of the 1,4naphthalenediphosphonic acid (28.2\% calculated) Fig. S6.

As a result, the use of structurally rigid 1,4-NDPA- $\mathrm{H}_{4}$ and changing the reaction conditions to optimize the binding modes and angles of the bridging ligands have provided a new platform to produce the porous MOPs. The addition of 4,4'-bpy helped engineer the copper coordination surface, restraining the copper coordination modes while adding extra bridging capacity into the metal organic frameworks. The structures reported has relatively higher BET surface areas for MOFs constructed using the organophosphonates and the first porous frameworks using short and structurally rigid aromatic organophosphonates. Adjustment of the angle between the bridging ligands 4,4'-bpy and 1,4-NDPA$\mathrm{H}_{4}$ is influential in the final pore sizes. Unlike the pillared layered structures produced with 1,4-phenyldiphosphonic acid, this strategy has produced microporous MOFs avoiding the formation of pillared compounds. The angular differences between the linear 4,4'-bpy and tetrahedral phosphonates could be further facilitated to produce engineered porous platforms in the future. In addition, the reported 8 membered one-dimensional chain structures could potentially be engineered to form predictable frameworks with increased tether arms. We have noted the chain structures in $\mathbf{1}$ and $\mathbf{2}$ as potential one dimensional secondary building units to form isoreticular expansions to obtain larger parallelogram and rectangular voids in this system. We are currently working on this hypothesis with longer aromatic organophosphonates.

\section{Supporting Information Summary}

The following files are available free of charge. The synthesis, crystallogprahic information, BET simulations and TGA.

\section{Acknowledgements}

Financial support by the Türkiye Bilimsel ve Teknolojik Araştırma Kurumu (TUBITAK 212T060) and COST action TD1304 are gratefully acknowledged.

Keywords: keyword 1 - keyword 2 - keyword $3 \cdot$ keyword 4 • keyword 5

[1] a) K. J. Gagnon, H. P. Perry, A. Clearfield, Chem. Rev. 2012, 112, 10341054; b) M. Taddei, F. Costantino, R. Vivani, Eur. J. Inorg. Chem. 2016 4300-4309.

[2] a) O. Yaghi, J. Am. Chem. Soc. 2016, 138, 15507-15509; b) O. M.Yaghi, M. O'Keeffe, N. W. Ockwig, H. K. Chae, M. Eddaoudi, J. Kim, Nature 2003, 423, 705-714.
[3] a) H.-C. Zhou, J. R. Long, O. M. Yaghi, Chem. Rev. 2012, 112, 673-674 b) H.-C. Zhou, S. Kitagawa, Chem. Soc. Rev. 2014, 43, 5415-5418; c) H. S. Cho, H. Deng, K. Miyasaka, Z. Dong, M. Cho, A. V. Neimark, J. K. Kang, O. M. Yaghi, O. Terasaki, Nature 2015, 527, 503-507; d) T. L. Easun, F. Moreau, Y. Yan, S. Yang, M. Schröder, Chem.Soc.Rev. 2017, 46, 239-274.

[4] a) D. Sahoo, R. Suriyanarayanan, V. Chandrasekhar, Dalton Trans. 2014, 43, 10898-10909; b) A. Schütrump, E. Kirpi, A. Bulut, F. L. Morel, M. Ranocchiari, E. Lork, Y. Zorlu, S. Grabowsky, G. Yücesan, J. Beckmann, Cryst. Growth Des. 2015, 15 (10), 4925-4931.

[5] a) A. Clearfield, Demadis K. D. in Metal Phosphonate Chemistry: From Synthesis to Applications, RSC Publishing, Cambridge, 2012; b) A. Bulut, Y. Zorlu, M. Wörle, S. Paşa, H. Kurt, J. Zubieta, J. Beckmann, G. Yücesan, Eur. J. Inorg. Chem. 2016, 3506-3512.

[6] a) J. M. Taylor, A. H. Mahmoudkhani, G. K. Shimizu, Angew. Chem. Int. Ed. 2007, 46(5), 795-798; b) M. Taddei, F. Costantino, F. Marmottini, A Comotti, P. Sozzani, R. Vivani, ChemComm 2014, 50, 14831-14834; c) M. Taddei, F. Costantino, R. Vivani, S. Sabatini, S. H. Lim, S. M. Cohen, ChemComm 2014, 50, 5737-5740; d) A. Bulut, Y. Zorlu, E. Kirpi, A. Cetinkaya, M. Wörle, J. Beckmann, G. Yücesan, Cryst. Growth Des. 2015, 15 (12), 5665-5669.

[7] a) N. B. Padalwar, K. J. Vidyasagar, Solid State Chem. 2016, 243, 83 94; b) R. Silbernagel, C. H. Martin, A. Clearfield, Inorg. Chem. 2016 , 55(4), 1651-1656; c) O. O. Ogunsolu, J. C. Wang, K. Hanson, ACS Appl. Mater. Interfaces 2015, 7(50), 27730-27734; d) T. L. Kinnibrugh, V. I. Bakhmutov, A. Clearfield, Cryst. Growth Des. 2014, 14(10), 4976-4984; e) P. O. Adelani, T. E. Albrecht-Schmitt, Cryst. Growth Des. 2012, 12(11), 5800-5805; f) P. O. Adelani, T. E. Albrecht-Schmitt, Angew. Chem. Int. Ed. 2010, 49(47), 8909-8911; g) P. O. Adelani, T. E. Albrecht-Schmitt, Inorg. Chem. 2009, 48(7), 2732-2734; h) W. Ouellette, G. Wang, H. Liu, G. T. Yee, C. J. O'Connor, J. Zubieta, Inorg. Chem. 2009, 48(3), 953963; i) J. M. Breen, W. Schmitt, Angew. Chem. Int. Ed. 2008, 47(36), 6904-6908; j) D.-K. Cao, S. Gao, L.-M. Zheng, J. Solid State Chem. 2004, 177(7), 2311-2315; k) B. Zhang, D. M. Poojary, A. Clearfield, Inorg. Chem. 1998, 37(8), 1844-1852; I) D. M. Poojary, B. Zhang, P. Bellinghausen, A. Clearfield, Inorg. Chem. 1996, 35(18), 5254-5263; m) P. O. Adelani, N. D. Cook, J.-M. Babo, P. C. Burns, Inorg. Chem. 2014, 53(8), 4169-4176; n) T. L. Kinnibrugh, N. Garcia, A. Clearfield, J. Solid State Chem. 2012, 187, 149-158; o) E. Brunet, H. M. H. Alhendawi, C. Cerro, M. J. de la Mata, O. Juanes, J. C. Rodriguez-Ubis, Angew. Chem. Int. Ed. 2006, 45(41), 6918-6920.

[8] a) H. Li, M. Eddaoudi, M. O'Keeffe, O. M. Yaghi, Nature 1999, 402, 276279; b) M. Eddaoudi, J. Ki, N. Rosi, D. Vodak, J. Wachter, M. O'Keeffe, O. M. Yaghi, Science 2002, 295(5554), 469-47.

[9] a) N. L. Rosi, J. Eckert, M. Eddaoudi, D. T. Vodak, J. Kim, M. O'Keeffe, O. M. Yaghi, Science 2003, 300(5622), 1127-1129; b) J. I. Feldblyum, A. G. Wong-Foy, A. J. Matzger, Chem. Commun. 2012, 48, 9828-9830.

[10] D. T. Vodak, M. E. Braun, J. Kim, M. Eddaoudi, O. M. Yaghi, Chem. Commun. 2001, 2534-2535.

[11] a) M. J. Bialek, J. Janczak, J. Zon, CrystEngComm 2013, 15(2), 390399; b) J. M. Breen, R. Clerac, L. Zhang, S. M. Cloonan, E. Kennedy, M. Feeney, T. McCabe, D. C. Williams, W. Schmitt, Dalton Trans. 2012, 41 2918-2926.

[12] S. Brunauer, P. H. Emmett, E. Teller, J. Am. Chem. Soc. 1938, 60, 309319. 
Entry for the Table of Contents

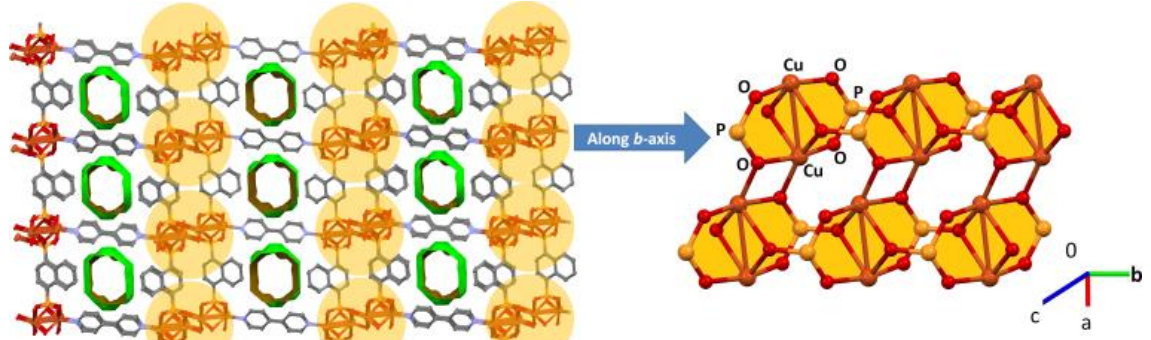

The 3D framework of 2 , showing the porosity of crystal, $1 \mathrm{D}-\mathrm{SBU}$ with edge sharing Cu-O-P-O- rings, and the central bridging $\left\{\mathrm{Cu}_{2} \mathrm{O}_{2}\right\}$ rhombi. 